\title{
Fault Location Algorithm for HVDC Transmission Based on Synchronized Fault Time
}

\author{
Kristianto Adi W. ${ }^{1}$, F. Danang Wijaya ${ }^{2}$, Tumiran ${ }^{3}$
}

\begin{abstract}
To determine the fault location of the de line fault in an HVDC transmission system, a new algorithm based on the traveling wave method and learning based method is proposed in this paper. The relationship between the traveling wave time of arrival differences with fault location is presented. The differences in traveling wave time of arrival measured at both ends of transmission line combined with associated fault locations form a fault pattern which is used to perform a simple calculation in order to determine the disturbance location. The fault current for different fault locations is simulated using the electromagnetic transient simulation software EMTDC/PSCAD. Performance of the proposed fault location algorithm is investigated using various fault location and resistance. The impact of data sampling rate also being investigated here. The simulation result shows that the proposed algorithm can reduce the sampling frequency and the number of train feature with the same accuracy.
\end{abstract}

Keyword-HVDC, Transmission, Fault Location, Traveling Wave, Double-end.

\section{INTRODUCTION}

Nowadays, HVDC transmission has been widely used as modern power transmission due to its advantages. HVDC has a large power transfer capability, longer transmission distance, lower losses, and can be utilized for accommodating very large intermittent electrical energy (i.e. solar and wind) [1], [2]. Passing complex terrain and operating under harsh climate condition make HVDC transmission susceptible to a fault Finding fault location is essential to shorten the outage time.

Most of the works about fault location have been proposed so far are based on traveling wave and few based on others like the distribution characteristic of distributed parameter transmission line model [3]. Traveling wave based method is divided into two groups with respect to the measurement technique. First is single-ended which only require one point of measurement and second is double-end which need two-point measurement at both ends of the transmission line [4].

Traveling wave method estimate the fault location based on the time needed by the wave generated by the fault to propagate to the end of the transmission line. This method has high precision and not affected by transmission condition such as loading state. Even though this method has high accuracy, the

${ }^{1}$ Student, Department of Electrical Engineering and Information Technology, Faculty of Engineering, Universitas Gadjah Mada, Jalan Grafika 2 Yogyakarta 44281 (phone: 0274-552305; email: kristianto.adi.w@mail.ugm.ac.id)

${ }^{2,3}$ Lecturer; Department of Electrical Engineering and Information Technology, Faculty of Engineering, Universitas Gadjah Mada, Jalan Grafika 2 Yogyakarta 55281 (phone: 0274-552305; email:danangwijaya@ugm.ac.id,tumiran@ugm.ac.id) reliability depends on the accuracy of detecting the head wave arrival time. The secondary reflecting wave head needs to be detected in the single-end method. This wave reflection is more difficult to detect than the first wave because the wave energy is reduced drastically. On the contrary, double-end method does not need to detect the reflection wave but it needs synchronous time measurement [5]. Normally double-end method is more accurate then single-end method. In addition, the traveling wave base method also requires the velocity of wave propagation. This velocity is varies corresponding to the line parameters, which have an effect on accuracy [1]. The wave propagation velocity can be obtained using a high sampling rate data acquisition device.

The work reported in [5] make use of a double-end traveling wave based method with unsynchronized data. It used a very high sampling frequency of $1 \mathrm{MHz}$ to calculate the fault location. The single-end traveling wave based method also had been proposed in [2], [6], these methods needed a sampling frequency of $80 \mathrm{kHz}$ and $>100 \mathrm{kHz}$ sequentially.

Another alternative to calculating fault location was introduced. Fault location is predicted base on the machine learning technique [2], [7], [8]. This method has promising appropriate performance and good flexibility in different condition. The important point in machine learning based method is to determine the suitable input feature and select the best match algorithm in accordance to solve the problem. The accuracy of machine learning based method is affected by adequate training data. A large set of training data can increase the accuracy, nevertheless, large training data cause a longer training time.

In this paper, a combination of double-end traveling wave based method and machine learning based method are proposed for fault location calculation in HVDC transmission system in order to reduce data sampling frequency. In this proposed algorithm, the velocity of traveling wave propagation in the transmission line is not used in the calculation process. The differences in traveling wave time of arrival measured at both ends of the transmission line are used as a feature to form a fault pattern. Fault pattern is a combination between the differences of traveling wave time of arrival and fault distance measured from the measurement point. The fault location is calculated based on a fault pattern which is previously constructed.

The remainder of this paper is organized as follow. Section II, the basic fundamental of double-end traveling wave and the main concept of this algorithm are explained. Section III, fault pattern and test case are generated through simulating various fault with a different parameter in the bipolar HVDC model. Then the proposed algorithm is applied to the data and the result is analyzed in Section IV. Finally, the conclusion of the work presented in Section V. 


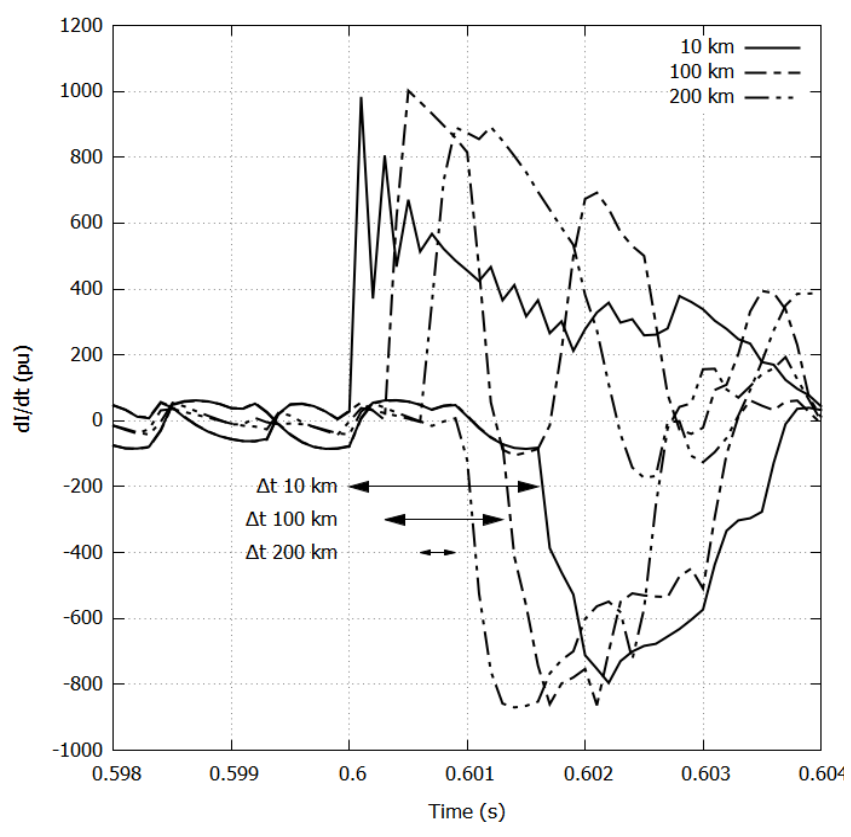

Fig. 1 Traveling wave time of arrival differences on several fault locations.

\section{CONCEPT AND MAIN IDEA}

\section{A. Fault Pattern}

Fault on a transmission line generates a traveling wave of voltage and current. These traveling waves will travel from the fault location point to both ends of the transmission line and reflected by transmission end and fault point (shown in Fig. 1). The arrival time of this wave can be used to calculate the exact disturbance location. In the double-end traveling wave method, only the first of wave head is used to calculate the fault location while the second wave head and others can be neglected.

Modern fault locator based on traveling wave uses a common time references for capturing the traveling wave time of arrival and communication link to exchange the information. Fault location can be calculated as follows.

$$
d=\frac{1}{2}\left(L+\left(t_{r 1}-t_{i 1}\right) \times v\right)
$$

where $L$ is the total of transmission line length, $t_{r 1}$ is the traveling wave time of arrival measured at R point, $t_{i 1}$ is the traveling wave time of arrival measured at I point, $v$ is the propagation velocity of traveling wave [9].

Suppose the propagation velocity of traveling wave in (1) considered to be constant, then the fault location only depends on the traveling wave time of arrival difference since the length of transmission line is also constant. The difference in traveling wave time of arrival reaches the lowest value when a fault occurs in $\mathrm{R}$ terminal or when fault distance is zero, this is due to the traveling wave in $\mathrm{R}$ terminal can be detected directly when a fault occurs, but traveling wave need time to propagate to the I terminal before it detected there thus the value of time arrival differences will be a negative value. The value of traveling wave time of arrival difference becomes zero when a disturbance occurs right in the middle of the transmission line.

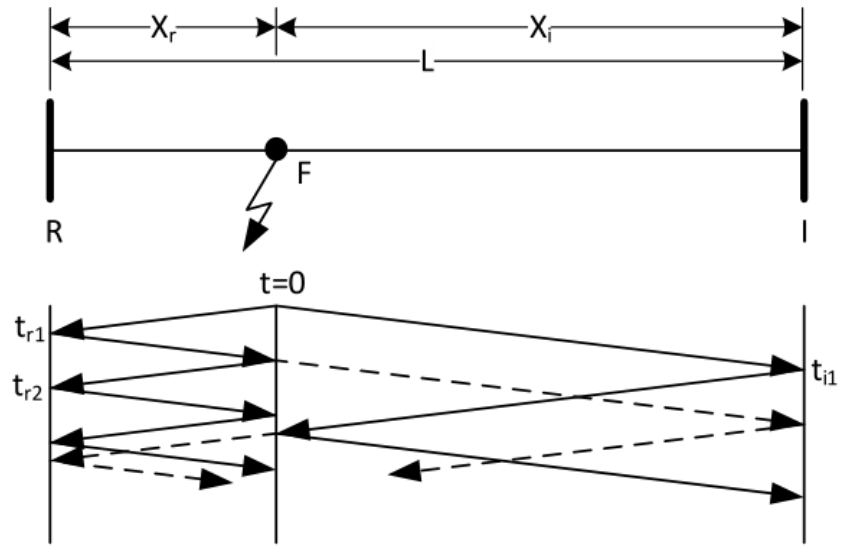

Fig. 2 Traveling wave phenomenon on transmission line.

When a fault occurs in terminal $\mathrm{I}$, the value of the difference in traveling wave time of arrival becomes maximal, this event is the opposite to fault that happens in R terminal. Another pair of data fault location and traveling wave time of arrival difference can be obtained using simulation as shown in Fig. 2. The value of time difference increase when a fault location getting further from the starting point of distance measurement. With these pair of data which form a fault pattern, the fault location can be calculated by utilizing input traveling wave time of arrival difference.

\section{B. Fault Location Algorithm}

The signals from the current are measured at both ends of the dc transmission line. The measuring device is installed in front of the smoothing reactor. Before executing the proposed fault location method, disturbance in HVDC transmission must be detected and classified. There is some reported research about detection and classification that can be used to detect and distinguish the fault type in [10], [11]. The discussion about the detection of a fault and its classification is not included in this study.

After fault detection and classification being conducted, the current signals which have been recorded in the memory are used to acquire the traveling wave time of arrival differences. The method to acquire traveling wave time of arrival is adopted from [9]. When the absolute value of the current differential exceeds the threshold, time correspondent to this event is written. It then compared with the time of disturbance that measured at another end of the transmission line. This process is keep repeated for another different fault location along the transmission line. The result of these comparisons will form a pattern in the form of the relationship between traveling wave arrival of time difference and fault distance.

This pattern is different for each type of fault. Therefore, the fault classification is needed to choose a suitable pattern for calculating fault location. For example, when positive to ground fault occurs the pattern of fault time differences in the positive pole is selected to use in fault locating. This rule also applies to two other disturbance type. Fault location then can be calculated using this following equation. 


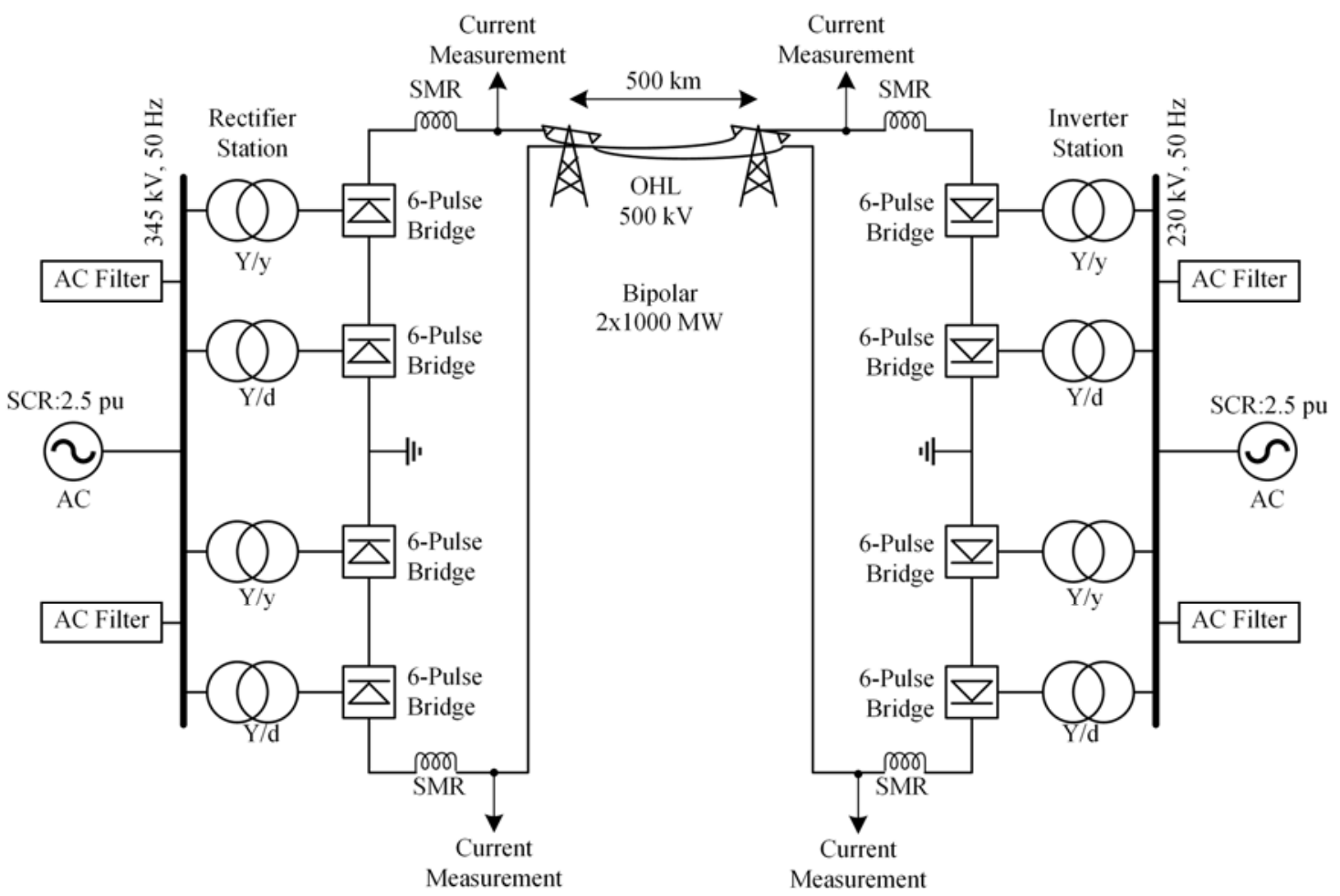

Fig. 3 Schematic diagram of HVDC transmission model under this study.

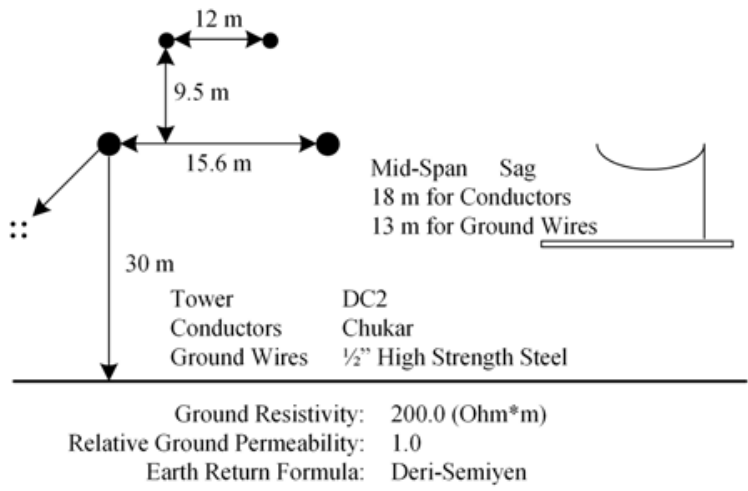

Fig. 4 The configuration of the transmission line and its tower.

$$
\begin{aligned}
d^{\prime}= & \frac{\sum_{i=1}^{n} d_{i} \times \sum_{i=1}^{n} t_{i}{ }^{2}-\sum_{i=1}^{n} t_{i} \times \sum_{i=1}^{n} t_{i} d_{i}}{n \times \sum_{i=1}^{n} t_{i}^{2}-\left(\sum_{i=1}^{n} t_{i}\right)^{2}} \\
& +\frac{n \times \sum_{i=1}^{n} t_{i} d_{i}-\sum_{i=1}^{n} t_{i} \times \sum_{i=1}^{n} d_{i}}{n \times \sum_{i=1}^{n} t_{i}^{2}-\left(\sum_{i=1}^{n} t_{i}\right)^{2}} \times t^{\prime}
\end{aligned}
$$

where $\left(t_{1}, d_{1}\right),\left(t_{2}, d_{2}\right) \cdots\left(t_{n}, d_{n}\right)$ are pattern relationship between fault time difference and fault distance, $t^{\prime}$ is fault time differences, and $d^{\prime}$ is fault location.

In short, the proposed algorithm can be explained using these following steps.

1. Record current signal during fault and find fault type according to the fault signal.

2. Determining the time where the absolute value of the current differential in the faulted pole exceeds the threshold value. Compare this time with the time from another end of the transmission line.
3. Generating new pattern using extracted current samples.

4. Calculate the fault location corresponding to the new pattern using (2).

\section{EXPERIMENTAL SETUP}

\section{A. Experiment Mode}

A model was established in the electromagnetic transient simulation software EMTDC/PSCAD for fault simulation in the HVDC transmission system. This model is based on CIGRE HVDC benchmark model [12] with a few modifications, especially on the transmission section. The capacity of rectifier and inverter stations are 1,000 MVA. The AC system on the rectifier side has a voltage rating of $345 \mathrm{kV}, 50 \mathrm{~Hz}$ and the $\mathrm{AC}$ system on the inverter side has a voltage rating of $230 \mathrm{kV}, 50$ $\mathrm{Hz}$. DC transmission voltage rating is $500 \mathrm{kV}$ with nominal power of 2,000 MW [13]. Fig. 3 shows one-line diagram of this model.

The total length of Over Head Line (OHL) used in this model is $500 \mathrm{~km}$. OHL is simulated by employing the frequency dependent model. Fig. 4 displays configuration of OHL and its tower. Detail configurations of OHL are as follow. It consists of two bundle conductors and two ground wire. Every bundle conductor consists of four wire. The structure of the tower and OHL in this model are adopted from [5].

\section{B. Generating Fault Location Pattern and Test Data}

Data for fault pattern and test are obtained from various simulation using the system model by varying the fault type, location, resistance, and data sampling rate. These data are generated based on various disturbance type such as positive to ground, negative to ground, and positive to negative. 
Simulation condition for acquiring data pattern are as follows.

- Fault distance along the transmission line varies from 10 to $490 \mathrm{~km}$ with a step of $5 \mathrm{~km}$.

- Values of fault resistance are $0.01,10$, and $100 \Omega$.

- The frequency for sampling data takes values of 40,20, and $10 \mathrm{kHz}$.

Also, simulation conditions for test data are as follows.

- Fault occurs at 15 different random locations.

- Fault resistance take value of 1, 10, 100, 200, and $400 \Omega$.

- Frequency sampling data are 40,20, and $10 \mathrm{kHz}$

Fault location pattern and test data are created using the method presented in section II-B step 1 until 3. Since the fault samples are taken every $5 \mathrm{~km}$ with 3 different fault resistance, it can be understood that each generated pattern consists of 291 combinations of traveling wave time of arrival difference and fault location.

\section{RESUltS AND ANALYSIS}

The performance index of this proposed method being calculated using (3) and also considered as percentage error of fault location estimation.

$$
\varepsilon_{r}=\frac{\left|d_{e s t}-d_{a c t}\right|}{L} \times 100 \%
$$

where $d_{\text {est }}$ is the calculated fault location measured from the rectifier station, $d_{\text {act }}$ is the exact fault location measured from the rectifier station, and $L$ is the total length of the dc transmission line.

The proposed algorithm is tested to apprehend the performance of the algorithm. Testing of the proposed algorithm is divided into several scenarios. These scenarios are as follows.

1. Testing the proposed algorithm with constant frequency sampling data $40 \mathrm{kHz}$ and fixed fault resistance of $1 \Omega$

2. Testing with constant frequency sampling data of $40 \mathrm{kHz}$ and variation of fault resistance $(1,10,100,200$, and 400 $\Omega)$.

3. Testing with the variation of frequency sampling data and constant fault resistance of $1 \Omega$.

\section{A. Fault Location Calculation Result}

The purpose of testing using scenario 1 is to understand the ability of the proposed algorithm to calculate various fault location. The results of this experiment are used as basic performance to be compared with the effect of changes in fault resistance and sampling frequency. The location of the disturbance in each type is calculated to get the estimated fault location.

Based on the condition state at III-B, each fault type has 15 test pattern for fault distance. Table I present the calculation result of various fault distance measured from rectifier station including the percentage of error. As can see from this table the absolute average percentage error of positive to a ground fault, negative to ground fault and positive to a negative fault are $0.213 \%, 0.249 \%$, and $0.157 \%$ respectively. These error values are under $1 \%$, which means these values are still in an acceptable range.
The average errors for fault type positive to ground and negative to ground are more than positive to negative type. It means that in positive to ground and negative to ground fault cases, the pattern is less accurate to represent fault location. If the features to establish the pattern are extended by considering adding more fault location with the nearly small step variations, it is expected to achieve a more accurate pattern. However, by adding more feature, it will extend the time which is required for simulation and construction of the fault pattern.

Another method to increase test result accuracy is to raise the sampling frequency. This technique can increase the pattern accuracy furthermore it can increase test result accuracy. By increasing the sampling frequency, it means that more sophisticated hardware is needed which leads to a less economical fault locator system.

\section{B. Effect of Fault Resistance}

The idea of the test using scenario 2 is to investigate the influence of fault resistance in test result accuracy. The test results of this scenario are compared with the results of testing from scenario 1 to measure how significant the sampling frequency impacts on the accuracy.

The calculation of fault location is being carried out to 15 test sample. The minimal, maximal, and average from error results of calculation fault location are presented in Table II. The fault resistance effect on calculation fault location especially in negative to ground fault. Small fault resistance shows a small impact on calculation accuracy and so the high fault resistance shows a greater impact on accuracy. According to the test result, the absolute average percentage error of all fault type are under $1 \%$. These errors are still in an acceptable range.

The accuracy decline when calculating fault with high fault resistance. Fault resistance influence the fault current, more high fault resistance, the smaller the magnitude of fault current. This can cause difficulty in detecting the time when disturbance occur. To overcome this problem, the sensitivity of the time detector must be recalibrated to accommodate the declining of the magnitude current fault due to high fault resistance.

\section{Effect of Sampling Rate}

In this section, the effect of different frequency to fault location calculation accuracy is studied through varying the sampling frequency of the current signal. The proposed algorithm is applied in the current signal and the maximum, minimum, and average percentage error for various sampling frequency are shown in Table III.

The percentage error of calculation result increase when the sampling frequency is reduced. The average percentage error as a result of fault location calculation with a sampling frequency of $20 \mathrm{kHz}$ for three type fault is still in acceptable range. On the contrary, the average percentage error of fault location calculation with a sampling frequency of $10 \mathrm{kHz}$ is higher the acceptable range. The proposed fault location algorithm still can be applied with a sampling frequency of 20 $\mathrm{kHz}$ with the percentage error slightly higher than sampling frequency of $40 \mathrm{kHz}$ but it still in the tolerable range. 
TABLE I

CALCULATION RESUlts FOR DifFERENT FAULT TYPE AND LOCATION

\begin{tabular}{|c|r|r|r|r|r|r|}
\hline \multirow{2}{*}{ Actual (km) } & \multicolumn{2}{|c|}{ Positive Ground Fault } & \multicolumn{2}{c|}{ Negative Ground Fault } & \multicolumn{2}{c|}{ Positive Negative Fault } \\
\cline { 2 - 7 } & Est. (km) & \multicolumn{1}{c|}{ Error } & Est. (km) & \multicolumn{1}{c|}{ Error } & \multicolumn{1}{c|}{ Est. (km) } & \multicolumn{1}{c|}{ Error } \\
\hline 82 & 83.736 & $0.35 \%$ & 83.741 & $0.35 \%$ & 83.274 & $0.26 \%$ \\
\hline 89 & 89.682 & $0.14 \%$ & 89.684 & $0.14 \%$ & 89.221 & $0.04 \%$ \\
\hline 98 & 98.601 & $0.12 \%$ & 98.6 & $0.12 \%$ & 98.141 & $0.03 \%$ \\
\hline 100 & 101.574 & $0.32 \%$ & 101.572 & $0.31 \%$ & 101.114 & $0.22 \%$ \\
\hline 113 & 113.466 & $0.09 \%$ & 113.459 & $0.09 \%$ & 113.008 & $0 \%$ \\
\hline 127 & 128.331 & $0.27 \%$ & 128.318 & $0.26 \%$ & 127.875 & $0.18 \%$ \\
\hline 141 & 143.196 & $0.44 \%$ & 143.177 & $0.44 \%$ & 142.742 & $0.35 \%$ \\
\hline 226 & 226.441 & $0.09 \%$ & 226.389 & $0.08 \%$ & 225.998 & $0 \%$ \\
\hline 271 & 271.036 & $0.01 \%$ & 270.966 & $0.01 \%$ & 270.599 & $0.08 \%$ \\
\hline 290 & 291.847 & $0.37 \%$ & 291.769 & $0.35 \%$ & 291.413 & $0.28 \%$ \\
\hline 302 & 303.739 & $0.35 \%$ & 303.656 & $0.33 \%$ & 303.307 & $0.26 \%$ \\
\hline 373 & 372.119 & $0.18 \%$ & 372.009 & $0.20 \%$ & 371.696 & $0.26 \%$ \\
\hline 392 & 392.93 & $0.19 \%$ & 392.811 & $0.16 \%$ & 392.51 & $0.10 \%$ \\
\hline 416 & 416.714 & $0.14 \%$ & 419.558 & $0.71 \%$ & 416.297 & $0.06 \%$ \\
\hline 471 & 470.228 & $0.15 \%$ & 470.079 & $0.18 \%$ & 469.818 & $0.24 \%$ \\
\hline Max & & $0.439 \%$ & & $0.712 \%$ & & $0.348 \%$ \\
\hline Min & & $0.007 \%$ & & $0.007 \%$ & & $0 \%$ \\
\hline Average & & $0.213 \%$ & & 0.249 & & $0.157 \%$ \\
\hline
\end{tabular}

TABLE II

CAlCulation Result Ffr Different Fault Type AND Fault Resistance

\begin{tabular}{|c|c|c|c|c|c|c|c|c|c|}
\hline \multirow{2}{*}{ Resistance $(\Omega)$} & \multicolumn{3}{|c|}{ Positive Ground Fault } & \multicolumn{3}{|c|}{ Negative Ground Fault } & \multicolumn{3}{|c|}{ Positive Negative Fault } \\
\hline & Max & Min & Average & Max & Min & Average & Max & Min & Average \\
\hline 1 & $0.44 \%$ & $0.01 \%$ & $0.21 \%$ & $0.71 \%$ & $0.01 \%$ & $0.25 \%$ & $0.35 \%$ & $0 \%$ & $0.16 \%$ \\
\hline 10 & $0.44 \%$ & $0.01 \%$ & $0.21 \%$ & $0.71 \%$ & $0.01 \%$ & $0.26 \%$ & $0.35 \%$ & $0 \%$ & $0.16 \%$ \\
\hline 100 & $0.74 \%$ & $0.09 \%$ & $0.33 \%$ & $1.03 \%$ & $0.08 \%$ & $0.34 \%$ & $0.35 \%$ & $0 \%$ & $0.16 \%$ \\
\hline 200 & $1.03 \%$ & $0.09 \%$ & $0.38 \%$ & $0.44 \%$ & $0.01 \%$ & $0.21 \%$ & $0.35 \%$ & $0 \%$ & $0.16 \%$ \\
\hline 400 & $0.44 \%$ & $0.01 \%$ & $0.21 \%$ & $1.86 \%$ & $0.12 \%$ & $0.57 \%$ & $0.65 \%$ & $0 \%$ & $0.20 \%$ \\
\hline
\end{tabular}

TABLE III

CALCUlation RESUlt For DifFERENT FAUlt TyPE AND SAMPLING FREQUENCY

\begin{tabular}{|c|c|c|r|r|r|r|r|r|r|}
\hline \multirow{2}{*}{ Frequency (kHz) } & \multicolumn{3}{|c|}{ Positive Ground Fault } & \multicolumn{3}{c|}{ Negative Ground Fault } & \multicolumn{3}{c|}{ Positive Negative Fault } \\
\cline { 2 - 10 } & Max & Min & Average & Max & \multicolumn{1}{c|}{ Min } & Average & Max & \multicolumn{1}{c|}{ Min } & Average \\
\hline 40 & $0.44 \%$ & $0.01 \%$ & $0.21 \%$ & $0.44 \%$ & $0.01 \%$ & $0.21 \%$ & $0.35 \%$ & $0 \%$ & $0.16 \%$ \\
\hline 20 & $1.59 \%$ & $0.17 \%$ & $0.64 \%$ & $1.58 \%$ & $0.17 \%$ & $0.64 \%$ & $1.11 \%$ & $0.04 \%$ & $0.66 \%$ \\
\hline 10 & $2.49 \%$ & $0.18 \%$ & $1.44 \%$ & $2.48 \%$ & $0.28 \%$ & $1.53 \%$ & $2.70 \%$ & $0.28 \%$ & $1.13 \%$ \\
\hline
\end{tabular}

Detail of effect fault resistance and sampling frequency to average percentage error can be observed trough histogram in Fig. 5. The proposed method has the best performance to a wide range of fault resistance with the sampling frequency of 40 $\mathrm{kHz}$. The utilization of the sampling frequency of $20 \mathrm{kHz}$ showed good accuracy in wide range fault resistance only in positive to negative disturbance type. The average percentage of error in this type of fault in an acceptable range which is bellow 1\%. Different from positive to negative fault type, the utilization of sampling frequency of $20 \mathrm{kHz}$ in two other fault type only limited to fault resistance with value $200 \Omega$. This error can be reduced by adding more fault feature to fault pattern. This mean increases the simulation and calculation time to prepare fault pattern. The error averages for fault location calculation using a sampling frequency of $10 \mathrm{kHz}$ are more than $1 \%$ for every fault resistance. This implies that a sampling frequency of $10 \mathrm{kHz}$ and a fault pattern generated from every $5 \mathrm{~km}$ are lacking in calculate fault location. The accuracy of calculation using sampling frequency of $10 \mathrm{kHz}$ can be improved by adding fault feature in the fault pattern. However, this action can prolong the training time.

\section{Comparison with Other Works}

This proposed algorithm only uses $40 \mathrm{kHz}$ for sampling frequency and training features from fault for every $5 \mathrm{~km}$ to generate a fault pattern. It is different from genuine traveling wave based fault location algorithm which usually needs high sampling frequency such as $1 \mathrm{MHz}$ as shown in [5]. Some study utilizes signal processing technique to reduce the sampling frequency like shown in [1] which used $100 \mathrm{kHz}$ for the sampling rate. This comparative is required to test whether the proposed method can degrade the sampling rate without decrease the accuracy. 


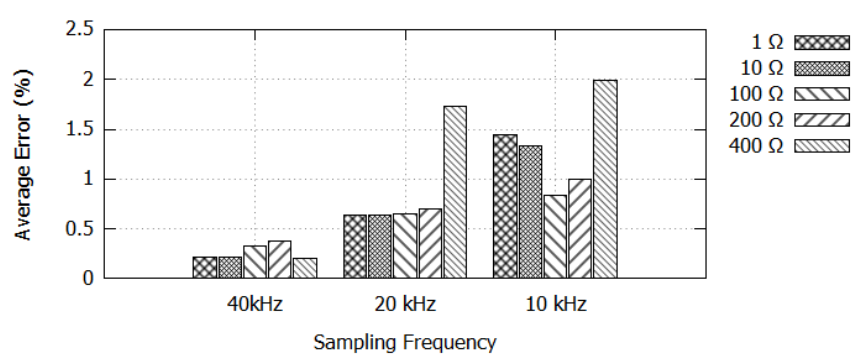

(a)

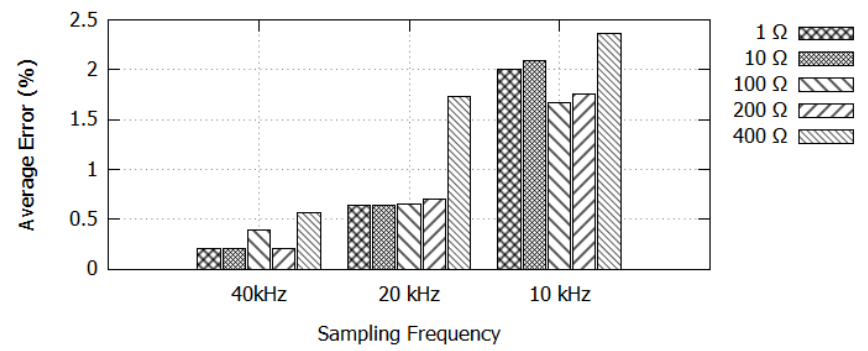

(b)

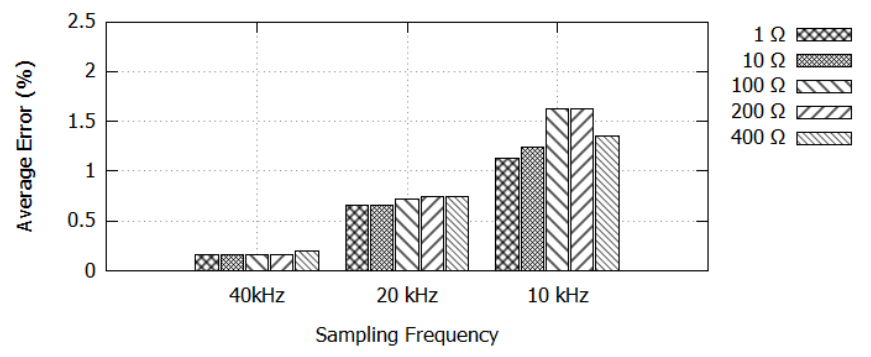

(c)

Fig. 5 Average percentage errors for different fault type with influence of fault resistance and sampling frequency, (a) positive to ground, (b) negative to ground fault, (c) positive to negative fault.

In order to deliver a fair comparative test, the proposed algorithm and method [5] and [1] which are compared adopt the same parameter model. Average percentage error in each method is analyzed with a statistical test. This comparison uses the significance level or $\alpha$ of $5 \%$ with the main hypothesis is the average percentage error from all methods are the same. The asymptotic significance results using Kruskal-Wallis for comparison with [5] and using independent t-test for comparison with [1] are 0.133 and 0.024 respectively. These results determine that proposed algorithm and method [5] has the same accuracy with the significance level of $5 \%$. The proposed algorithm has different accuracy with method in [1] with a significance level of $5 \%$. The average of error proposed method is $0.077 \%$ lower than method in [1]. These results confirm that the proposed method can reduce the frequency sampling rate without degrading the result accuracy.

Another comparison is conducted to methods based on a combination of traveling wave and machine learning. The accuracy of the algorithm in this method depends on the number of feature to train and frequency sampling data. The purpose of this comparison is to test whether the proposed method can reduce the number of features and frequency sampling data without reducing accuracy.
Study on the utilization of wavelet transform to decompose traveling wave to use as training feature in Support Vector Regression (SVR) in order to predict fault location in HVDC transmission line was reported in [2]. This method used a sampling rate of $>100 \mathrm{kHz}$ with 1,062 training feature. Another research use SVR to predict fault location was described in [7]. This research used time delay, characteristic frequency, energy attenuation, and high-frequency energy obtained from the decomposition of traveling wave with Hilbert-Huang Transform (HHT) as training feature to SVR. Training features were constructed from a fault occurred for every $5 \mathrm{~km}$ along the transmission line with a sampling frequency of $1 \mathrm{Mhz}$. The asymptotic significance results using Kruskal-Wallis for comparison with [2] and independent t-test for comparison with [7] are 0.166 and 0.290 respectively. It can be concluded that the proposed algorithm has the same accuracy as method in [2] and in [7] with a significance level of 5\%. This comparison result verifies that the proposed method can use lower training feature with the same accuracy in calculating the fault location.

\section{CONCLUSION}

In this paper, an algorithm to solve the fault location calculation in the HVDC transmission line based on a combination of double-end traveling wave method and learning-based method is proposed. It can clearly understand that there is a rule between fault distance and the differences in traveling wave time of arrival measured at both ends of the transmission line. The relationship between fault distance and the differences in traveling wave time of arrival has been investigated. An HVDC based on CIGRE benchmark model build in EMTDC/PSCAD is used to study this relationship and gives a satisfactory result with a wide variation of fault type, fault location, and high fault resistance. A fault pattern generated by a low sampling frequency of $40 \mathrm{kHz}$ can be utilized to calculate fault location with average errors for fault type of positive ground, negative ground, and positive-negative are $0.213 \%, 0.249 \%$, and $0.157 \%$ respectively.

The comparison study with another traveling wave based method shows that the proposed method can reduce the frequency sampling without reducing the accuracy result with a significance level of 5\%. Even in some case, the proposed method can improve accuracy. Compared with a method based on a combination of traveling wave and machine learning, this proposed method show able to maintain the accuracy even with the reduction of sampling rate and the total number of training feature with a significance level of $5 \%$. In summary, the algorithm presented in this paper can reduce the data sampling rate and the total number of training feature significantly compared to the ordinary traveling wave based method or combination of traveling wave and machine learning based method.

\section{REFERENCES}

[1] Z. He, K. Liao, X. Li, S. Lin, J. Yang, and R. Mai, "Natural FrequencyBased Line Fault Location in HVDC Lines," IEEE Transactions on Power Delivery, Vol. 29, No. 2, pp. 851-859, April 2014.

[2] H. Livani and C.Y. Evrenosoglu, "A Single-Ended Fault Location Method for Segmented HVDC Transmission Line," Electric Power Systems Research, Vol. 107, pp. 190 - 198, 2014. 
[3] J. Suonan, S. Gao, G. Song, Z. Jiao, and X. Kang, "A Novel FaultLocation Method for HVDC Transmission Lines," IEEE Transactions on Power Delivery, Vol. 25, No. 2, pp. 1203-1209, April 2010.

[4] C. Li and P. He, "Fault-Location Method for HVDC Transmission Lines Based on Phase Frequency Characteristics," IET Generation, Transmission \& Distribution, Vol. 12, No. 4, pp. 912-916, 2018.

[5] J.M. Johnson and A. Yadav, "Complete Protection Scheme for Fault Detection, Classification, and Location Estimation in HVDC Transmission Lines Using Support Vector Machines," IET Science, Measurement \& Technology, Vol. 11, No. 3, pp. 279-287, 2017.

[6] L. Shang, G. Herold, J. Jaeger, R. Krebs, and A. Kumar, "High-Speed Fault Identification and Protection for HVDC Line Using Wavelet Technique," 2001 IEEE Porto Power Tech Proceedings, 2001, pp. 1-5.

[7] Y. Hao, Q. Wang, Y. Li, and W. Song, "An Intelligent Algorithm for Fault Location on VSC-HVDC System," International Journal of Electrical Power \& Energy Systems, Vol. 94, pp. 116 - 123, 2018.

[8] M. Farshad and J. Sadeh, "A Novel Fault-Location Method for HVDC Transmission Lines Based on Similarity Measure of Voltage Signals," IEEE Transactions on Power Delivery, Vol. 28, No. 4, pp. 2483-2490, Oct. 2013.
[9] E.O. Schweitzer, A. Guzman, M.V. Mynam, V. Skendzic, B. Kasztenny, and S. Marx, "Protective Relays with Traveling Wave Technology Revolutionize Fault Locating," IEEE Power and Energy Magazine, Vol. 14, No. 2, pp. 114-120, March-April 2016.

[10] Y. Zhang, N. Tai, and B. Xu, "Fault Analysis and Traveling-Wave Protection Scheme for Bipolar HVDC Lines," IEEE Transactions on Power Delivery, Vol. 27, No. 3, pp. 1583-1591, July 2012.

[11] S. Deb and H.K. Verma, "Selection of Fault Discriminators for Unit Protection of Monopolar HVDC Transmission System," 2017 3rd International Conference on Condition Assessment Techniques in Electrical Systems (CATCON), 2017, pp. 20-25.

[12] M. Szechtman, T. Margaard, J.P. Bowles, C.V. Thio, D. Woodford, Wess, R. Joetten, G. Liss, M. Rashwan, P.C. Krishnayya, P. Pavlinec, V. Kovalev, K. Maier, J. Gleadow, J.L. Haddock, N. Kaul, R. Bunch, R. Johnson, G. Dellepiane, and N. Vovos, "The Cigre HVDC Benchmark Model - A New Proposal With Revised Parameters," Electra, Vol. 157, pp. 61-66, Dec 1994.

[13] D. Kwon, H. Moon, R. Kim, C. Kim, and S. Moon, "Modeling of CIGRE Benchmark HVDC System Using PSS/E Compared with PSCAD," 2015 5th International Youth Conference on Energy (IYCE), 2015, pp. 1-8. 